\title{
Acute Necrotizing Encephalitis in an Adult Patient with Novel Influenza A (H1N1) Infection
}

\author{
Sulaiman alkhashan* and Nermeen Samir \\ Department of Neurology, Farawanyia Hospital, Kuwait
}

Submission: October 03, 2015; Published: October 17, 2015

*Corresponding author: Sulaiman alkhashan, Department of Neurology, Farawanyia Hospital, Kuwait; Email: ss_alkhashan@yahoo.com

\begin{abstract}
Neurologic complications of pandemic (H1N1) 2009 were first reported in children a case series of 4 pediatric patients in the United States in May 2009 in May 2009 [1]. Most subsequent reports were also of cases in children, because those <17 years of age appear most vulnerable [2], we are presenting a case of Encephalopathy in an adult young female with H1N1 influenza infection, with complete recovery, according to our records, this is the only case reported in the middle east .
\end{abstract}

\section{Introduction}

Influenza A (H1N1), which is also called human influenza virus with swine origin, has created a major worldwide health problem within a short time after its emergence [3]. This infection is often self- limited but sometimes can cause severe and fatal complications [4,5]. Involvement of the CNS in influenza virus infection is very rare, but serious manifestations like seizures, encephalitis, myelitis, Reye syndrome, and other neurologic disorders have been described previously in association with respiratory tract infection with seasonal influenza $\mathrm{A}$ or $\mathrm{B}$ viruses [6]. These findings indicate that, as with seasonal influenza, neurologic complications can occur with ongoing novel influenza A (H1N1) pandemic, but the frequency with which these occur is unknown. Encephalitis has been reported with novel H1N1 infection, mainly in children [7].

Other Neurologic manifestations of influenza are now known to include acute disseminated encephalomyelitis, GuillainBarre syndrome, transverse myelitis, and acute necrotizing encephalopathy (ANE). Reports of ANE began surfacing from Japan during the influenza epidemics of the mid-and late 1990s [1]. According to studies from Japan, in the most severe cases of influenza-associated ANE, patients develop altered mental status with or without seizures and then rapidly progress to a comatose state within a mean of 24-72 hours from the onset of fever and upper respiratory symptoms $[1,8,9]$. Seizures are often resistant to antiepileptic medications. Death, which occurs in roughly $30 \%$ of cases, results largely from cardiorespiratory compromise or complications from mechanical ventilation [10].

\section{Review of literature}

H1N1 a localized necrotizing encephalopathy is being increasingly recognized CNS complication of H1N1\& many case reports mainly from pediatric groups being published with few reported cases of adult patients with H1N1 encephalities IN 2012 ElSevier Medical journal published an H1N1 influenza associated encephalitis [3] one case was Adult Patient with Novel H1N1 Infection Presented with Encephalitis, Rhabdomyolysis, Pneumonia and Polyneuropathy [11]. Report of two rare complications of pandemic influenza A (H1N1) in November 2010 [12] which was aseptic meningitis and myocarditis. One reported case shows direct viral invasion of the CSF\& detection of viral RNA \& pleocytosis [10].

According to Joosten et al. [3] all patients were previously healthy, aged between 20 and 46 years, five were male and one was female. The most frequent initial clinical manifestations were influenza-like symptoms. Neurological symptoms included drowsiness, memory disturbance, disorientation, confusion, tremors and focal signs starting between 1 and 6 days after onset of illness. All patients had a laboratory-confirmed (nasopharyngeal swab) H1N1 virus infection. However, like in our patient, H1N1 RNA was not detected in CSF by RT-PCR. Other findings of CSF included elevated leukocyte counts and/ or elevated protein levels. Neuroimaging findings were variable ranging from normal to cortical and subcortical lesions, like in our patient, to involvement of deep brain structures with or without brain edema. All patients were treated with Oseltamivir. Two patients received simultaneously treatment with corticosteroids. There was a complete recovery of neurologic manifestations in 


\section{Open Access Journal of Neurology \& Neurosurgery}

two patients; in three other patients mild to severe neurologic sequelae were noted.

\section{Case Study}

To our knowledge, encephalopathy associated with the novel H1N1 influenza strain was first reported in a case series of 4 pediatric patients in the United States in May 2009 [13,14]. All 4 patients had mild seizures and/or altered mental status and all recovered fully without any neurological sequelae at discharge. There has been only few cases reported of encephalopathy in a novel H1N1 infection in adult; we are reporting a similar case in adult previously healthy female.

A 40 years old Indian female previously healthy was admitted with 3 days of medium grade fever, non productive cough, shortness of breath and was diagnosed with Pneumonia, her Neurological examination was within normal, Laboratory studies revealed increased white blood cell count $\left(17.4 \times 10^{9} / \mathrm{L}\right)$, Neutrophilia $85.5 \%$, decreased lymphocytes 6.0 , and elevated C-reactive protein of $15.8 \mathrm{mg} / \mathrm{dL}$. Serum electrolytes and renal and liver function tests were within normal limits. Her chest $\mathrm{x}$ ray shows bilateral scattered pneumonic patches; her H1N1 serology was positive, Tamiflu started on 3rd day of admission, Patient was shifted to intensive care unit and intubated Secondary to respiratory distress \&hypoxia. She was sedated by continuous Dormicum infusion \& Remifentanil, chest condition was getting better after three days from Tamiflu started.

Neurology service was consulted on day 6 as patient doesn't regain consciousness after discontinuation of sedation. $0 / \mathrm{E}$ :pt was unconscious, not responding to verbal stimulation, in response to pain there is extension of lower limbs \& moving upper limb, reflexes was brisk bilaterally, bilateral extensor planter Response. She had MRI, MRV, MRA done subsequently and showed Right frontal, right posterior parieto-occipital, left
Tempro - parieto - occipital, extensive high cerebral and patchy left upper cerebral as well as right thalamic extending to upper right midbrain: hyper intensity suggesting micro bleeds and hemorrhagic components in $\mathrm{T}_{2}$ with some restriction in DWI, some $\mathrm{T}_{1}$ hyper intensities serpentine signals are seen filling the related sulci and leptomeninges, likely representing meningeal exudates (Figure 1).

A lumbar puncture was performed. The CSF was clear colorless with normal Glucose \& protein levels with no cells, further workup to exclude other possible causes of encephalitis included (1) CSF polymerase chain reaction (PCR) for neurotropic virus was negative, including RT-PCR for 2009 H1N1 virus; (2) Cultures of blood, urine, tracheobronchial aspirate and CSF were negative; (3) Serology for mycoplasma pneumonia, Chlamydia, Rickettsia, hepatitis B and C, syphilis and HIV antibody was negative; (4) Testing for autoimmune disorders was within normal, absence of other causes and consistency of finding with other reported cases of the literature recommended the diagnosis of $\mathrm{H} 1 \mathrm{n} 1$ encephalitis . MRI brain follow up done after 40 days shows: Mild increase in size and signal intensity abnormalities of the previously noted Rt frontal, Rt posterior parieto-occipital, left temporo-parietoOccipital, extensive Rt cerebellar and patchy upper cerebellar as well as Rt. thalamic extending to the upper right midbrain swollen edematous gyri and follia.

A course of IVIG was tried based on the reported cases of autoimmune nature of the disease, $2 \mathrm{gm} / \mathrm{Kg}$ over 5 days, after which patient shows much improvement \& regained consciousness 2 days after last dose of IVIG. Patient was is fully conscious, oriented to time, place \& persons, indulges in family conversation, no focal neurological signs, apart from generalized weakness, preserved peripheral reflexes, bilateral equivocal planter responses, with no visual field defects, no signs of cranial nerves affection, preserved gag response, she was on nasogastric

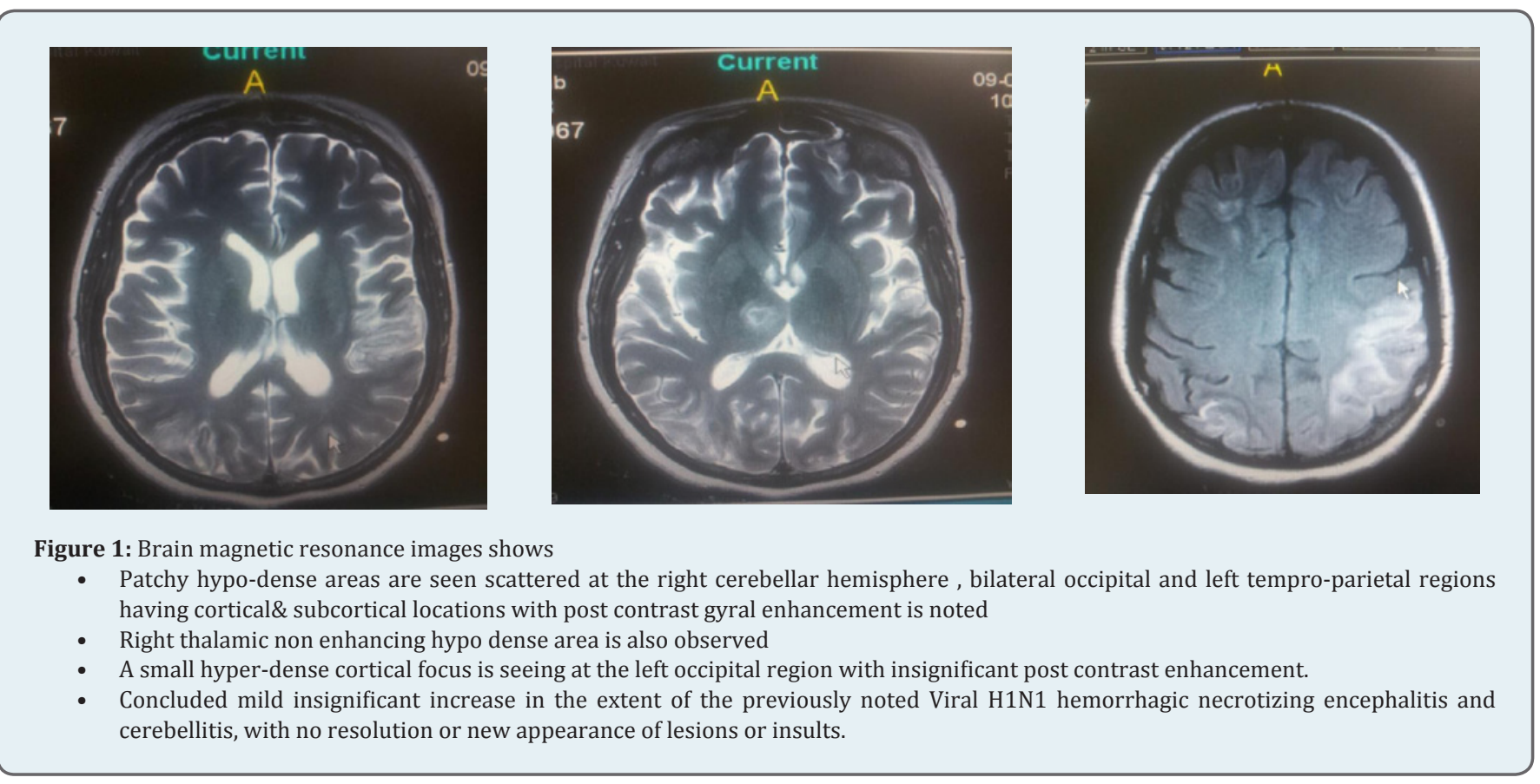




\section{Open Access Journal of Neurology \& Neurosurgery}

Table 1(a,b): Clinicopathologic features of previously reported cases of H1N1 influenza-associated encephalopathy/encephalitis in adults.

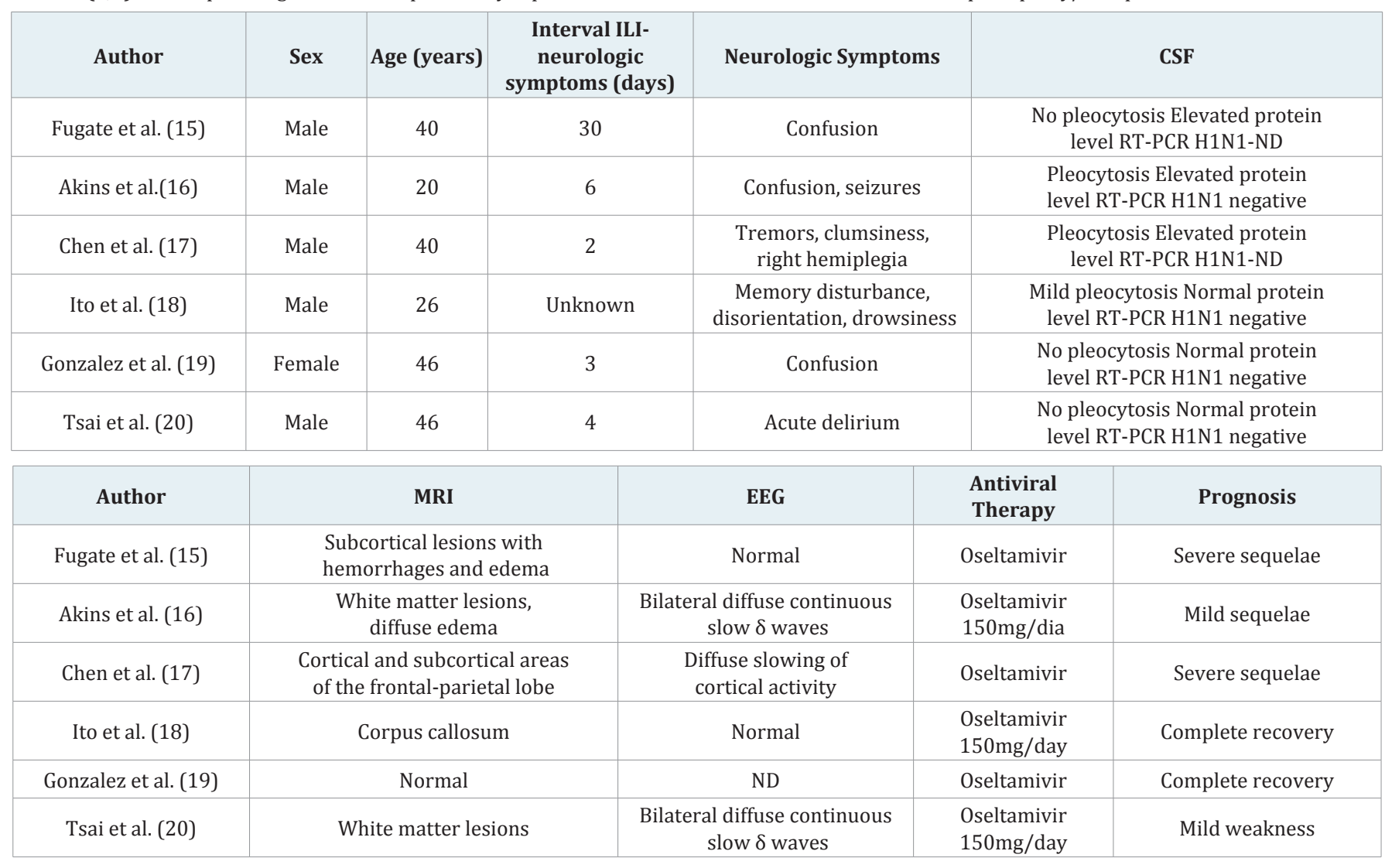

ND: Not Done; ILI: Influenza-Like Illness; CSF: Cerebrospinal Fluid; EEG: Electroencephalography.

tube feeding till she regain consciousness \& started oral feeding. She was started on physical Rehab, but she was found to have bilateral drop foot with no back pain, preserved peripheral reflexes; on EMG showed: Generalized extensive axonal damage suggesting critical illness neuropathy, she was discharged after 35 days (Table 1 ) [15-20] .

\section{Discussion}

H1N1-associated encephalitis was defined by the Center of Disease Control and Prevention as altered mental status $>24 \mathrm{~h}$, in patients with laboratory-confirmed H1N1 virus infection, within 5 days of influenza-like illness symptom onset plus two or more of the following: fever, focal neurological signs, CSF pleocytosis, EEG and/or abnormal neuroimaging indicative of encephalitis [1]. Our patient almost fulfilled all of these criteria. However it is noteworthy that neurological signs and symptoms were noted almost 20 days after the initial onset of respiratory illness when sedation was discontinued.

The clinical course of ANE is rapidly progressive; patients present with fever and nonspecific symptoms, such as cough, emesis, and/or diarrhea, and quickly develop neurologic dysfunction. Approximately $18 \%$ of cases of ANE in Japan have been associated with influenza A infection [3]. The strain most frequently associated with ANE is influenza A, H3N2 subtype, although cases associated with $\mathrm{H} 1 \mathrm{~N} 1$ and influenza B has also been described [2,7,11-13]. The disease is associated with significant morbidity and mortality, and survivors usually exhibit at least short-term neurologic sequelae [6]. In addition to antiviral therapies such as Oseltamivir, corticosteroids and intravenous immune globulin have been used to treat selected cases of ANE in Japan, with varying degrees of patient improvement [12]. Currently, there is no definitive treatment for ANE, and management of these patients' centers upon supportive care for neurologic failure and treatment of increased intracranial pressure if present. Then they start monitoring some of Acute necrotizing Encephalitis in H1N1 adult infection.

\section{References}

1. Centers for Disease Control and Prevention (2009) Neurologic complications associated with novel influenza A (H1N1) virus infection in children-Dallas, Texas, May 2009. MMWR Morb Mortal Wkly Rep 58(28): 773-778.

2. Yildizdaş D, Kendirli T, Arslanköylü AE, Horoz OO, Incecik F, et al. (2011) Neurological complications of pandemic influenza (H1N1) in children. Eur J Pediatr 170(6): 779-788.

3. Joosten A, Moya B, Nunes J, Germano N, Alcântara J, Bento L (2012) ânidade de UrgênciaMédica - Hospital São José, Centro HospitalarLisboa Central, Lisbon, Portugal, Elsevier 36: 520-522.

4. Zimmer SM, Burke DS (2009) Historical Perspective - Emergence of Influenza A (H1N1) viruses. N Engl J Med 361(3): 279-285.

5. Jain S, Kamimoto L, Bramley AM, Schmitz AM, Benoit SR, et al. (2009) Hospitalized patients with 2009 H1N1 Influenza in the United States, 


\section{Open Access Journal of Neurology \& Neurosurgery}

April-June 2009. N Engl J Med 361(120): 1935-1944.

6. Nickel CH, Stephan FP, Dangel M, Blume K, Gehrisch R, et al. (2009) First wave of the influenza A/H1N1v pandemic in Switzerland. Swiss Med Wkly 139(51-52): 731-737.

7. Maricich SM, Neul JL, Lotze TE, Cazacu AC, Uyeki TM, et al. (2004) Neurologic complications associated with influenza A in children during the 2003-2004 influenza season in Houston, Texas. Pediatrics 114(5): e626-e633.

8. Togashi T, Matsuzono Y, Narita M, Morishima T (2004) Influenzaassociated acute encephalopathy in Japanese children in 1994-2002. Virus Res 103(1-2): 75-78.

9. Sugaya N (2002) Influenza-associated encephalopathy in Japan. Semin Pediatr Infect Dis 13(2): 79-84

10. Smidt MH,Stroink H, Bruinenberg JF, Peeters M (2004) Encephalopathy associated with influenza A. European Journal of Paediatric Neurology 8(5): 257-260.

11.http://cid.oxfordjournals.org/content/50/8/e50.full\#content-block

12. Aristine Cheng, Kuei-Hong Kuo, Chia-JuiYang (2011) Pandemic (H1N1) 2009 Encephalitis in Woman, Taiwan. Centers for disease control and prevention 17(10): 1-3.

13. Patel KK, Patel AK, Shah S, Ranjan R, Shah SV (2012) Adult Patient with a Novel H1N1 Infection Presented with Encephalitis, Rhabdomyolysis, Pneumonia and Polyneuropathy. J Glob Infect Dis 4(3): 178-81.

14. Babamahmoodi F1, Davoodi AR, Ghasemian R, Delavarian L (2012) Report of two rare complications of pandemic influenza A (H1N1). J Infect Dev Ctries 6(2): 204-207.

15. Fugate JE, Lam EM, Rabinstein AA, Wijdicks EF (2010) Acute hemorrhagic leukoencephalitis and hypoxic brain injury associated with H1N1 influenza. Arch Neurol 67(6): 756-758.

16. Akins PT, Belko J, Uyeki TM, Axelrod Y, Lee KK, et al. (2010) H1N1 encephalitis with malignant edema and review of neurologic complications from influenza. Neurocrit Care 13(3): 396-406.

17. Chen YC, Lo CP, Chang TP (2010) Novel influenza A (H1N1)-associated encephalopathy/encephalitis with severe neurological sequelae and unique image features - a case report. J Neurol Sci 298(1-2): 110-113.

18. Ito S, Shima S, Ueda A, Kawamura N, Asakura K, et al. (2011) Transient splenial lesion of the corpus callosum in H1N1 influenza virusassociated encephalitis/encephalopathy. Intern Med 50(8): 915-918.

19. Gonzalez BE, Brust DG (2009) Novel influenza A (H1N1) presenting as an acute febrile encephalopathy in a mother and daughter. Clin Infect Dis 49(12): 1966-1967.

20. Tsai CK, Lai YH, Yang FC, Chen CY, Peng GS (2011) Clinical and radiologic manifestations of $\mathrm{H} 1 \mathrm{~N} 1$ virus infection associated with neurological complications: a case report. Neurologist 17(4): 228-231. 